\title{
System Identification and Tuning of Wireless Power Transfer Systems with Multiple Magnetically Coupled Resonators
}

\author{
Johan Winges, Thomas Rylander, Carl Petersson, Christian Ekman, Lars-Åke Johansson and Tomas McKelvey
}

\begin{abstract}
We present a procedure for system identification and tuning of a wireless power transfer (WPT) system with four magnetically coupled resonators, where each resonator consists of a coil and a capacitor bank. The system-identification procedure involves three main steps: 1) individual measurement of the capacitor banks in the system; 2) measurement of the frequency-dependent two-port impedance matrix of the magnetically coupled resonators; and 3 ) determining the inductance of all coils and their corresponding coupling coefficients using a Bayesian approach. The Bayesian approach involves solving an optimization problem where we minimize the mismatch between the measured and simulated impedance matrix together with a penalization term that incorporates information from a direct measurement procedure of the inductance and losses of the coils. This identification procedure yields an accurate system model which we use to tune the four capacitance values to recover high system-performance and account for, e.g., manufacturing tolerances and coil displacement. For a prototype WPT system, we achieve $3.3 \mathrm{~kW}$ power transfer with $91 \%$ system efficiency over an air-gap distance of approximately $20 \mathrm{~cm}$.
\end{abstract}

Index Terms-Bayesian estimation, charging electric vehicles, impedance matching, magnetically coupled resonators, system identification, tuning, wireless power transfer (WPT).

\section{INTRODUCTION}

Magnetically coupled resonators are becoming increasingly popular for mid-range wireless power transfer (WPT) that is suitable for charging electric vehicles [1], [2], [3]. Given the low magnetic coupling that is typical in such WPT systems, resonant circuits with low losses are used to achieve high power transfer at high efficiency. However, it is crucial that such resonators are tuned in order to achieve satisfactory system performance, as the power transfer and efficiency rapidly decays should the system become de-tuned [4], [5].

Aldhaher et al. [6] studied a WPT system with two resonators, where they mitigated de-tuning issues associated with coil misalignment and displacement by means of a current-controlled inductor and a variable switching frequency. However, in automotive applications, it may not be possible

Manuscript accepted Januari 12, 2018. This work was funded by the Swedish Energy Agency in the project "Säker induktiv energiöverföring för elfordon - Safe Wireless Energy (SAWE)", which has the project number 38577-1.

J. Winges, T. Rylander and T. McKelvey are with the Department of Electrical Engineering, Chalmers University of Technology, Göteborg, 41296 Sweden (e-mail: winges@chalmers.se; rylander@chalmers.se).

C. Petersson, C. Ekman and L.-À. Johansson are with QRTECH AB, Flöjelbergsgatan 1c, Mölndal, 43135 Sweden.

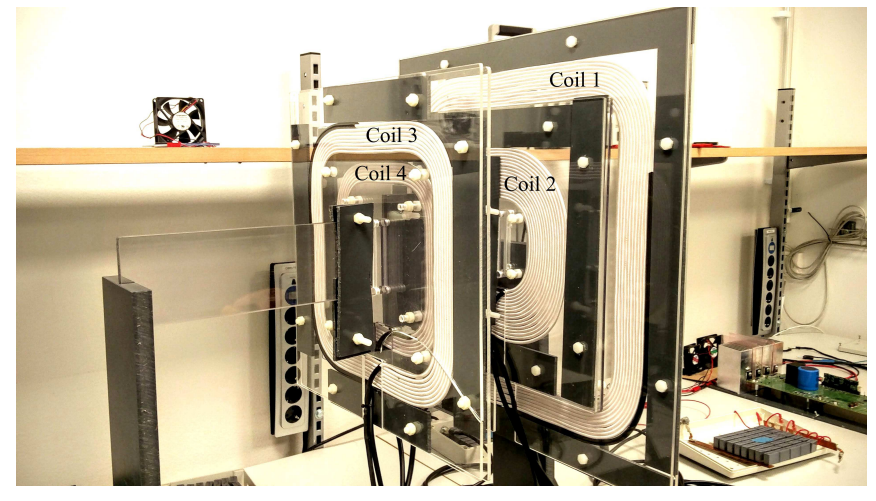

Fig. 1. Photo of the air-wound coils used in the WPT system prototype with four magnetically coupled resonators.

to change the switching frequency due to regulations and standards [7]. Beh et al. [8] considered a WPT system that operates at a fixed frequency $(13.56 \mathrm{MHz})$, where they used an automatized impedance matching system with three extra components (an L-match network) to tune the resonance frequency of the resonator pair to that of the power source. Similarly, Li et al. [9] used four tunable components for a WPT system with two resonators, which allows for some advantages, such as making the resonant network inductive and achieving zero-voltage switching. Halpern et al. [10] analyzed the limits of capacitor tuning for a WPT system with two resonators and present the optimal selection of the capacitance values with respect to maximizing a weighted sum of both system efficiency and power transfer.

In an effort to increase the power transfer distance and efficiency, WPT systems with more than two magnetically coupled resonators have been considered in the literature [11], [12], [13]. Zhong et al. [14] presents a wide variety of socalled domino WPT systems where a large number of magnetic resonators are placed in a domino pattern to increase the power transfer range. Liu et al. [15] show how two additional resonant circuits can be located at an off-center location about midway between the primary and secondary resonators to increase the range and performance of a WPT system. However, the total size of such domino-like WPT systems may be unpractical for some mid-range high power transfer applications such as electric vehicle charging. Lin et al. [16] show how it is possible to identify the capacitance values and inter coil distances for a 3 -coil domino system from measurements of the input voltage 
and current over a frequency band using a genetic optimization algorithm. However, in their work they assume that the coil losses and inductance can be accurately calculated, which is not necessarily the case for more involved coil designs.

In this extended article based on the conference submission [17], we present a system identification procedure combined with tuning for a WPT system with four magnetically coupled resonators, where two resonators are located at the primary side and two on the secondary side. Here, the four resonators can improve the efficiency and power transfer for applications with low magnetic coupling between the primary and secondary side [11], [12]. Moreover, the extra resonant circuits yields additional degrees of freedom in the WPT system design, and we show that that the capacitance values can be used for system tuning. In the system identification step, we estimate the coil inductances, mutual couplings and losses using a Bayesian approach [18], where we minimize the weighted sum of two terms: 1) the mismatch between the coil parameters and an approximate measurement of these parameters; and 2) the mismatch between the simulated and measured impedance matrix for the magnetically coupled resonators over a frequency band. Here, the system identifications is used to find deviations from a nominal system design due to e.g. manufacturing tolerances and coil misalignment. Furthermore, we demonstrate that high power transfer and efficiency can be recovered for an experimentally realized (non-nominal) design at a fixed operating frequency using only one tunable capacitor per resonant circuit, where the capacitance of each capacitor can be adjusted in a few discrete steps. In addition, the realized design complies with a set of circuit constraints which prevent over-heating or voltage breakdown of the components in the WPT system.

\section{WPT SYSTEM DESIGN}

A WPT system consisting of four magnetically coupled resonators is considered and Fig. 1 shows a photograph of the coils in a WPT system prototype. The coil geometry of the prototype is described in Table I in terms of the number of turns $N_{m}$, the side length $s_{m}$ and the placement along the $z$-axis, which is perpendicular to the plane of each coil and coincides with their respective centers. Here, the air gap between the two groups of coils is $18.5 \mathrm{~cm}$. We use Litz wire with an outer diameter of $10 \mathrm{~mm}$, consisting of 1080 copper strands with a diameter of $0.1 \mathrm{~mm}$. For all coils, the minimum inner bend radius is $4.5 \mathrm{~cm}$. The different coil sizes in the prototype system were chosen to illustrate that different geometrical constraints may be applicable to the primary and secondary side in a WPT system depending on the application. Further, the small additional coils may in theory be incorporated into the same plane as the large coils, which yields a WPT system that is relatively thin, which is important for e.g. charging electric vehicles.

We use COMSOL Multiphysics ${ }^{\circledR}[19]$ to compute the selfinductance $L_{m}$ of each coil and the mutual coupling coefficient $k_{m n}$ between each coil, and the results are presented in Table I. The individual Litz wires of the coils are not explicitly modeled in the COMSOL model, which instead uses a multiturn coil domain applied to a simplified coil geometry where we approximate the coil cross-section as a rectangle of height equal to the wire diameter and length equal to the number of turns times the wire diameter.

TABLE I

GEOMETRY DESCRIPTION WITH NUMBER OF TURNS $N_{m}$ OF LITZ WIRE

$(\oslash=10 \mathrm{~mm})$, OUTER SIDE LENGTH $s_{m}$ AND AXIAL POSITION $z_{m}$ OF THE COILS SHOWN IN FIG. 1 ENUMERATED BY $m=1, \ldots, 4$ AND

CORRESPONDING INDUCTANCE AND COUPLING COEFFICIENTS COMPUTED USING A COMSOL MODEL

\begin{tabular}{lllll}
\hline Coil $m$ & 1 & 2 & 3 & 4 \\
\hline$N_{m}(-)$ & 11 & 16 & 8 & 6 \\
$s_{m}(\mathrm{~cm})$ & 51.0 & 29.0 & 34.0 & 25.0 \\
$z_{m}(\mathrm{~cm})$ & 0 & 2.50 & 24.5 & 22.0 \\
\hline$L_{m}(\mu \mathrm{H})$ & 120 & 66.4 & 41.3 & 15.6 \\
$k_{1 m}(\%)$ & - & 21.3 & 9.09 & 6.87 \\
$k_{2 m}(\%)$ & 21.3 & - & 6.99 & 6.77 \\
$k_{3 m}(\%)$ & 9.09 & 6.99 & - & 37.9 \\
\hline
\end{tabular}

\section{A. Circuit model}

A circuit diagram model of the magnetically coupled resonators are shown in Fig. 2, which also includes capacitor banks and series resistances that model losses in the system. Our nominal system design for the prototype is described by the component values in Table I and the capacitance values $C_{1}=27.0 \mathrm{nF}, C_{2}=42.7 \mathrm{nF}, C_{3}=67.7 \mathrm{nF}$ and $C_{4}=140 \mathrm{nF}$.

At the terminals $\mathrm{A}_{1}-\mathrm{A}_{2}$, the magnetically coupled resonators are fed by a power inverter, which is modeled by a squarewave voltage source $u_{\text {in }}(t)=U_{0} \operatorname{sgn}\left[\cos \left(\omega_{\mathrm{p}} t\right)\right]$ in series with an internal resistance $R_{\mathrm{G}}=0.25 \Omega$. It operates at the frequency $f_{\mathrm{p}}=85 \mathrm{kHz}$ with a maximum output voltage of $U_{0}^{\max }=450 \mathrm{~V}$. At the terminals $B_{1}-B_{2}$, the magnetically coupled resonators are loaded by a rectifier, smoothing filter and resistive load $R_{\mathrm{L}}=27 \Omega$ as shown in Fig. 3. The current-voltage curve of the diodes is modeled as piece-wise linear with a forward voltage drop of $1.3 \mathrm{~V}$ and a forward resistance of $0.08 \Omega$. We define the efficiency $\eta$ of the system as the power $p_{\text {load }}$ transferred to the load $R_{\mathrm{L}}$ divided by the input power $p_{\text {in }}$ supplied by the generator. The power levels are found after the system has reached steady-state operation, and are time-averaged over one period. The complete circuit is simulated in time-domain using the circuit simulation software LTspice and an in-house circuit simulation implementation in MATLAB.

\section{SysteM IDENTIFICATION}

In simulation, the nominal system design presented in Section II achieves both high power transfer and efficiency. However, our experimental realization suffers from various manufacturing tolerances and, consequently, the actual component values of the magnetically coupled resonators differ from the nominal design. Such deviations are common in practical situations where misalignment and incorrect distance between 

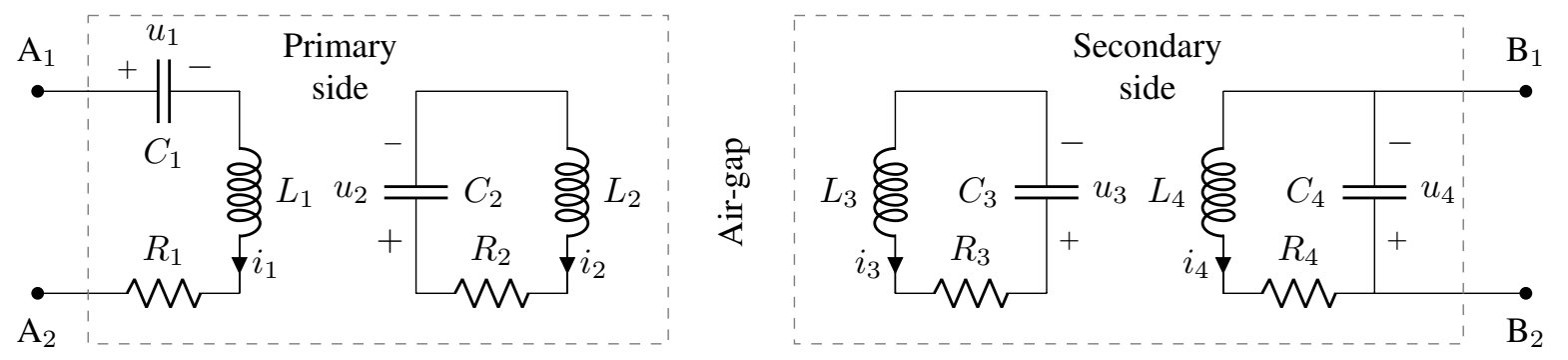

Fig. 2. Circuit diagram for the magnetically coupled resonators with four coils and capacitors.

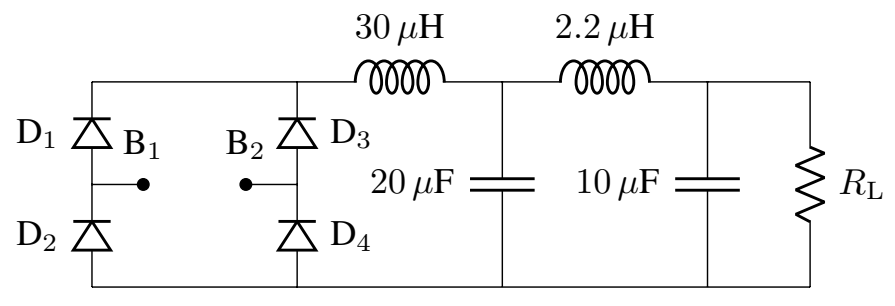

Fig. 3. Circuit diagram for rectifier, filter and load resistance.

the primary and secondary side are typical disturbances. Thus, we propose the following procedure for identifying the component parameters of WPT systems.

First, the capacitance of the capacitor banks is measured to high accuracy when they are disconnected from the rest of the circuit. Here, the capacitor banks are assumed to be tunable to a few discrete values, where we measure each discrete capacitance value to high accuracy.

Next, the realized system parameters $\mathbf{p}=\left[L_{1}, \ldots, L_{4}\right.$, $\left.k_{12}, \ldots, k_{34}, R_{1}, \ldots, R_{4}\right]$ of the magnetically coupled resonators are identified. Initially, the coil inductance, coupling coefficients and losses are measured approximately using a direct procedure where we disconnect the individual coils from the rest of the circuit. The approximate measurement result is denoted $\mathbf{p}_{0}$. Next, we connect the capacitor banks (which are set to known values) to form the system of the magnetically coupled resonators. The corresponding model is shown in Fig. 2 and we introduce the two-port impedance matrix

$$
\mathbf{Z}=\left[\begin{array}{ll}
Z_{A A} & Z_{A B} \\
Z_{B A} & Z_{B B}
\end{array}\right]
$$

where the index $A$ corresponds to the terminals $\mathrm{A}_{1}-\mathrm{A}_{2}$ and the index $B$ corresponds to the terminals $\mathrm{B}_{1}-\mathrm{B}_{2}$ and, due to reciprocity, we have $Z_{B A}=Z_{A B}$. The impedance matrix $\overline{\mathbf{Z}}\left(f_{\nu}\right)$ is then measured for $N_{\nu}$ frequency points in a frequency band around the operating frequency of the WPT system. To simplify the notation, we vectorize the three unique elements of the impedance matrix with respect to frequency, i.e. $\overline{\mathbf{z}}=\operatorname{vec}\left\{\bar{Z}_{A A}\left(f_{\nu}\right), \bar{Z}_{A B}\left(f_{\nu}\right), \bar{Z}_{B B}\left(f_{\nu}\right)\right\}$. (Here the bar notation indicates that the quantity is measured.)

To identify the system parameters $\mathbf{p}$, we use a Bayesian approach [18] to formulate a weighted maximum a posteriori estimator that combines: 1) the mismatch between the measured impedance matrix $\overline{\mathbf{z}}$ and the simulated impedance matrix $\mathbf{z}(\mathbf{p})=\operatorname{vec}\left\{Z_{A A}\left(f_{\nu}, \mathbf{p}\right), Z_{A B}\left(f_{\nu}, \mathbf{p}\right), Z_{B B}\left(f_{\nu}, \mathbf{p}\right)\right\}$; and 2) a penalization term that corresponds to the deviation between the parameters $\mathbf{p}$ subject to optimization and the measured parameters $\mathbf{p}_{0}$ from the direct procedure. This gives the minimization problem

$$
\min _{\mathbf{p}} \frac{1}{N_{\mathbf{z}}}\|\mathbf{z}(\mathbf{p})-\overline{\mathbf{z}}\|_{\mathbf{P}}^{2}+\frac{\gamma}{N_{p}}\left\|\mathbf{p}-\mathbf{p}_{0}\right\|_{\mathbf{Q}}^{2}
$$

We assume independent Gaussian distributions for the measurement errors and compute the norms with respect to the diagonal matrices $P_{k k}=1 /\left|\delta_{z_{k}} \bar{z}_{k}\right|^{2}$ and $Q_{l l}=1 /\left(\delta_{p_{l}} \bar{p}_{l}\right)^{2}$, where $\delta_{z_{k}}$ and $\delta_{p_{l}}$ are estimates of the relative standard deviation of the respective measurement uncertainties. In this work, we use $\delta_{z_{k}}=1 / 3 \%$ for $\overline{\mathbf{z}}$, and for the optimization parameters we use $\delta_{p_{l}}=1 / 3 \%$ for $L_{m}, \delta_{p_{l}}=2 \%$ for $k_{m n}$ and $\delta_{p_{l}}=5 \%$ for $R_{m}$. Furthermore, $N_{z}=3 N_{\nu}$ and $N_{p}=10$ are the number of elements in the vectors $\mathbf{z}$ and $\mathbf{p}$, respectively. From (2), we can interpret that the regularization parameter $\gamma$ penalizes deviation of the estimate $\mathbf{p}$ from the measurement $\mathbf{p}_{0}$, weighted by the corresponding measurement uncertainties of the direct measurement procedure.

We solve the minimization problem (2) using the gradientbased optimization algorithm fmincon implemented in MAT$L A B$ [20] with the initial guess $\mathbf{p}_{0}$, and an optimum is typically found in less than 70 iterations. An important feature of the proposed optimization problem (2) is that the impedance matrix model only involves linear components and can be solved efficiently in frequency domain. Consequently, the objective function is associated with a relatively low computational cost per frequency point and it is therefore feasible to resolve rapid frequency variations over a relatively large frequency band, which is beneficial for real-time applications.

\section{TUNING}

The system identification procedure in Section III yields accurate estimates of the circuit parameters, shown in Fig. 2, that deviate from the nominal design due to manufacturing tolerances and coil misalignment. Consequently, the realized system performance may have deteriorated considerably when compared to the nominal system. However, by tuning the four capacitance values, we can recover high power transfer and efficiency for rather significant perturbations due to, e.g., coil misalignment.

To recover high performance, we simulate the WPT system for a large set of different combinations of the discrete values of the capacitor banks and compare the final power transfer and 
TABLE II

CONSTRAINTS ON RMS VALUES OF THE CURRENTS AND VOLTAGES, WHICH ARE ENFORCED FOR THE COMPONENTS LISTED WITHIN THE PARENTHESES FOR $m=1, \ldots, 4$

\begin{tabular}{lrll}
\hline Component & Quantity & unit & max \\
\hline Coil $\left(L_{m}, R_{m}\right)$ & $i$ & $\mathrm{~A}$ & 60 \\
\hline Capacitor $\left(C_{m}\right)$ & $u$ & $\mathrm{kV}$ & 5 \\
& $i$ & $\mathrm{~A}$ & 40 \\
& $u \times i$ & $\mathrm{kVA}$ & 50 \\
\hline Power inverter $\left(R_{\mathrm{G}}\right)$ & $i$ & $\mathrm{~A}$ & 30 \\
\hline Rectifier $\left(\mathrm{D}_{m}\right)$ & $u$ & $\mathrm{~V}$ & 850 \\
& $i$ & $\mathrm{~A}$ & 15 \\
\hline
\end{tabular}

efficiency after the system has reached steady-state operation. For each of these solutions, we limit the generator voltage $U_{0}$ such that none of the constraints listed in Table II are violated. These constraints prevent over-heating or voltage breakdown for the particular components in the realized WPT prototype system. Here, we require that the power inverter is connected to an inductive load $Z_{\mathrm{AA}}^{\text {loaded }}$ when the terminals $\mathrm{B}_{1}-\mathrm{B}_{2}$ of the magnetically coupled resonators are connected to the load circuit shown in Fig. 3. Thus, we require that $0^{\circ}<\angle Z_{\mathrm{AA}}^{\text {loaded }}<90^{\circ}$ for the operational frequency $f_{\mathrm{p}}$ and remove all solutions that yield a non-inductive load.

The remaining solutions are sorted with respect to power transferred to the load $R_{\mathrm{L}}$ and a suitable candidate is selected.

\section{RESULTS}

We present results for the system identification and tuning of the WPT system shown in Fig. 1, which is a prototype system intended to transfer $3.3 \mathrm{~kW}$ power over a $20 \mathrm{~cm}$ air-gap at high efficiency. Prior to assembly, we measure all components with an OMICRON LAB Bode100 network analyzer using a direct procedure where each component is disconnected from the rest of circuit and measured. A rather limited set of discrete capacitance values for the capacitor banks are realized for the prototype system and the measured results are shown in Table III. The different capacitance values are implemented by a set of discrete low loss capacitors (Kemet $R 73$ series) in a parallel configuration connected by switches.

TABLE III

DisCRETE VALUES FOR EACH $C_{m}$ IN CAPACITOR BANKS

\begin{tabular}{ll}
\hline Parameter & Values \\
\hline$C_{1}(\mathrm{nF})$ & $17.6, \mathbf{2 1 . 7}, 26.4,30.8,35.2$ \\
$C_{2}(\mathrm{nF})$ & $23.5,31.1, \mathbf{3 9 . 2}, 47.0,54.8,62.7$ \\
$C_{3}(\mathrm{nF})$ & $47.0,53.7, \mathbf{6 2 . 0}, 66.4,73.9,80.6$ \\
$C_{4}(\mathrm{nF})$ & $110,122,130, \mathbf{1 3 9}, 145,152$ \\
\hline
\end{tabular}

Next, the coils and capacitors are assembled with the discrete values marked in bold in Table III to form the system of magnetically coupled resonators. We measure the impedance matrix $\overline{\mathbf{Z}}\left(f_{\nu}\right)$ for $N_{\nu}=600$ uniformly distributed frequency points $f_{\nu}$ from $50 \mathrm{kHz}$ to $170 \mathrm{kHz}$. In Fig. 4, we show the absolute value and phase of the measured impedance matrix $\bar{Z}_{p q}\left(f_{\nu}\right)$ with solid curves and the computed result using the coil parameters from the direct procedure, i.e. $Z_{p q}\left(f_{\nu}, \mathbf{p}_{0}\right)$, with dashed curves. It should be noted that there are substantial differences between the measured and computed results in Fig. 4, with an average relative error of about $40 \%$. This error indicates that we have an unsatisfactory identification of the component values in our system of magnetically coupled resonators. Here, this deviation is in part due to the measurement uncertainty of the direct measurement procedure in combination with incorrect inter-coil spacing which may occur as the WPT system is assembled prior to the impedance matrix measurement. It should be noted that the resonant behavior of the magnetically coupled resonators results in that the WPT system is relatively sensitive to rather small perturbations of the circuit parameters.

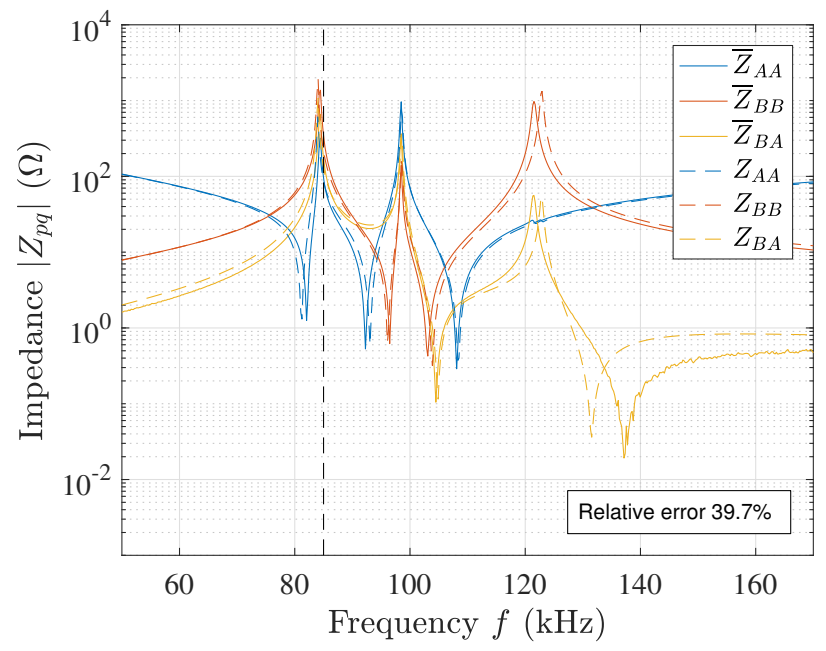

(a) Magnitude

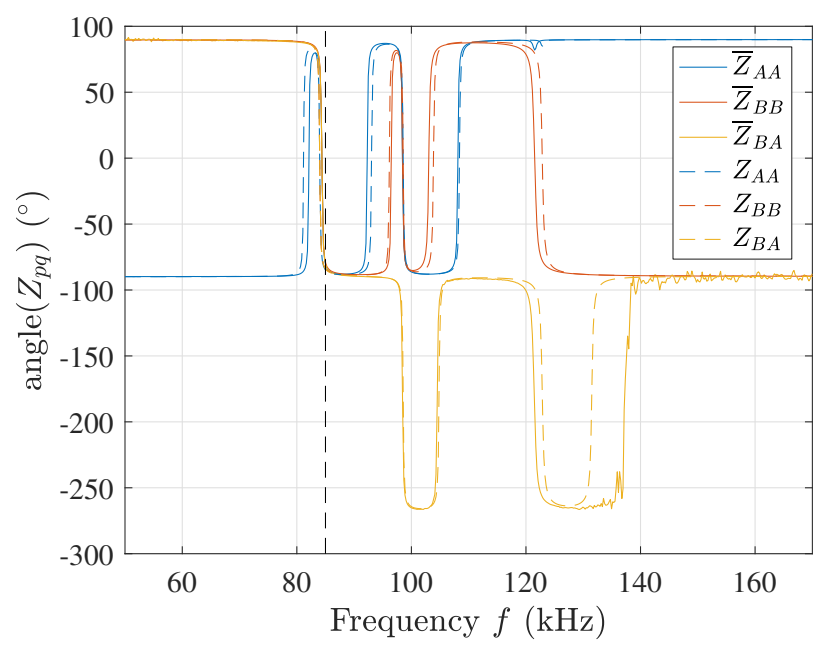

(b) Phase

Fig. 4. Magnitude and phase of impedance matrix entries as a function of frequency: 1) solid curves - measurements $\bar{Z}_{p q}\left(f_{\nu}\right)$; and 2) dashed curves model $Z_{p q}\left(f_{\nu}, \mathbf{p}_{0}\right)$ for the initial parameter vector $\mathbf{p}_{0}$. The vertical dashed line show the operating frequency $85 \mathrm{kHz}$. 


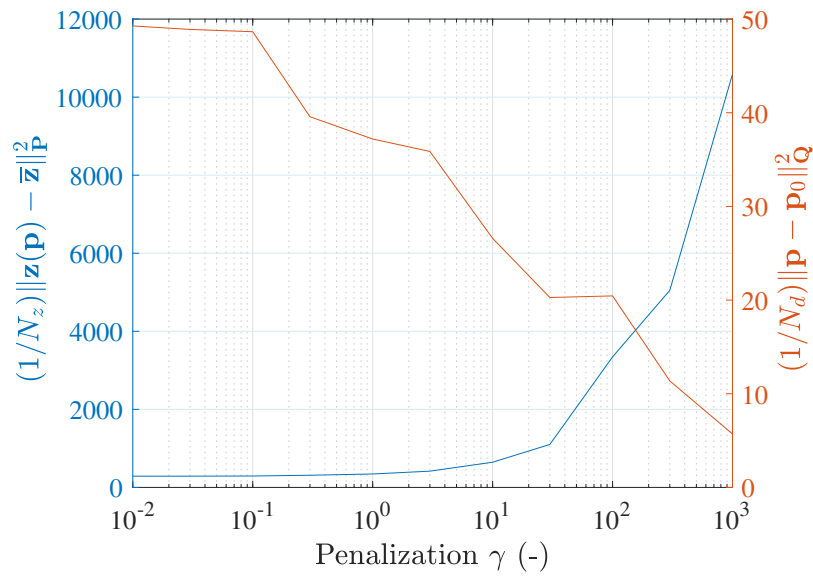

Fig. 5. Magnitude of the two different norms in the minimization problem (2) versus penalization.

We solve the optimization problem (2) for $10^{-2} \leq \gamma \leq 10^{3}$. Fig. 5 shows the corresponding magnitude of the two norms in (2) as a function of the penalization parameter $\gamma$. We note that the impedance matrix norm increases significantly for $\gamma>30$, which indicates that $\gamma=30$ is a suitable penalization value that achieves good model agreement, with low deviation between the optimization parameters and the measured parameters. Selecting the result with $\gamma=30$ yields the optimized parameter vector $\mathbf{p}^{*}$ and the corresponding impedance matrix entries $Z_{p q}\left(f_{\nu}, \mathbf{p}^{*}\right)$ which are shown with dashed curves in Fig. 6. For reference, we also show $\bar{Z}_{p q}\left(f_{\nu}\right)$ with solid curves in Fig. 6. It should be noted that the agreement is excellent after the system identification and that the average relative error is less than $4 \%$.

Table IV shows the initial parameter vector $\mathbf{p}_{0}$ and the optimized result $\mathbf{p}^{*}$. Here, we note a rather large reduction in the coupling coefficients associated with the air-gap when we compare the initial and optimized results. These results indicate that the distance between the primary and secondary side may have been increased by approximately $5-10 \mathrm{~mm}$ when the system was reassembled after the direct measurement of the individual components.

In realistic usage scenarios of WPT systems, air-gap deviations on the order of at least $10-20 \mathrm{~mm}$ are expected due to, among other factors, parking misalignment, variations in the ground clearance between different car models and installation differences between different charging stations. It is reassuring that the system identification procedure presented here significantly reduces the deviation between the model and the measurement. Thus, the complete WPT model presented in Section II can be used for accurate system simulation, which in turn can be used to tune the WPT system for high power transmission and efficiency.

Given the identified model parameters, we tune the four capacitor banks by comparing the simulated performance of the 1080 different discrete capacitance combinations given by Table III using an exhaustive search. For each combination in the exhaustive search, the generator voltage is reduced from $U_{0}^{\max }=450 \mathrm{~V}$ until all constraints in Table II hold and, consequently, the maximum power transfer can vary

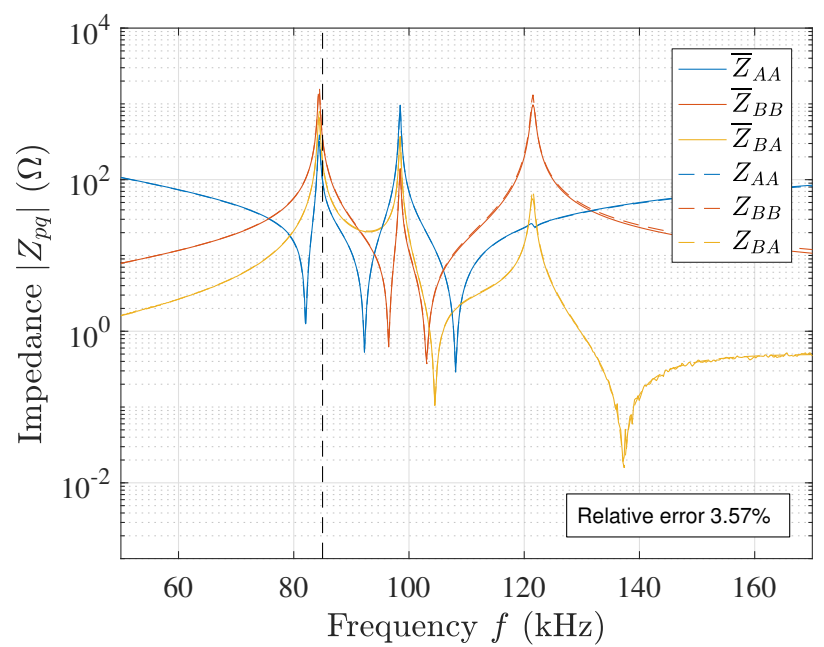

(a) Magnitude

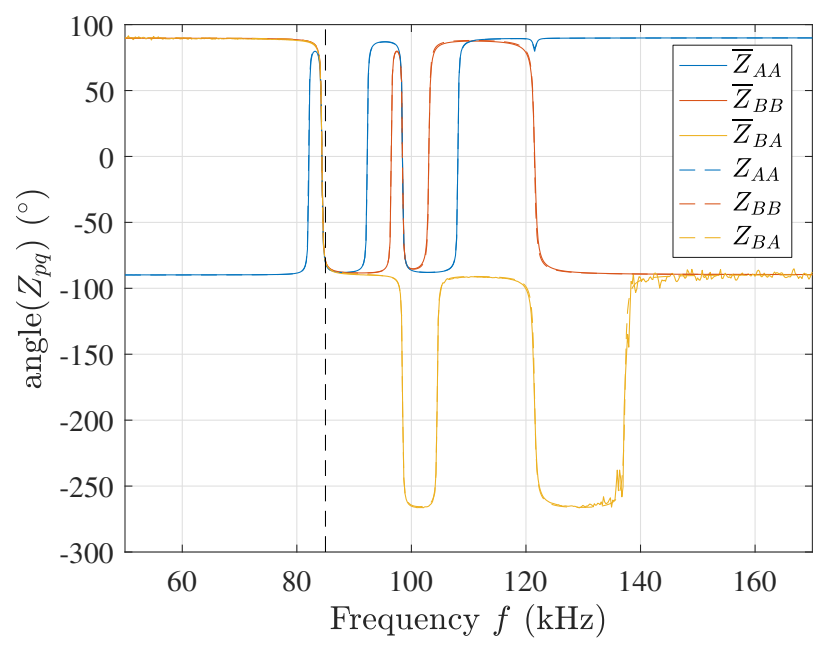

(b) Phase

Fig. 6. Magnitude and phase of impedance matrix entries as a function of frequency: 1) solid curves - measurements $\bar{Z}_{p q}\left(f_{\nu}\right)$; and 2) dashed curves model $Z_{p q}\left(f_{\nu}, \mathbf{p}^{*}\right)$ for the optimized parameter vector $\mathbf{p}^{*}$.

significantly between different realizations. In the search, we also identify and disregard capacitance combinations which yield non-inductive loading of the power inverter. For the prototype, more than 100 different capacitance combinations yielded a power transfer of at least $3.0 \mathrm{~kW}$ with system efficiency of above $87 \%$ in simulation. The top ten capacitance combinations with respect to maximum power transfer are presented in Table V.

The capacitance combination $C_{1}=26.4 \mathrm{nF}, C_{2}=39.2 \mathrm{nF}$, $C_{3}=62.0 \mathrm{nF}$ and $C_{4}=139 \mathrm{nF}$ marked in bold in Table V is selected as an appropriate tuning for the prototype system as it has good performance and only required that the first capacitance bank was switched from the previously used value of $C_{1}=21.7 \mathrm{nF}$. In an experiment that aim at a power transfer of $3.3 \mathrm{~kW}$, our prototype system transfers $3.35 \mathrm{~kW}$ at $91.4 \%$ efficiency with this capacitance selection. This results compares well with the expected power transfer of $3.49 \mathrm{~kW}$ at $91.1 \%$ efficiency predicted by the model at the lower generator input power used in the experiment. 
TABLE IV

INITIAL COMPONENT VALUES WHEN MEASURED INDIVIDUALLY FOR EACH COMPONENT BY A DIRECT PROCEDURE AND OPTIMIZED PARAMETER VALUES USING SYSTEM IDENTIFICATION PROCEDURE FOR WPT SYSTEM SHOWN IN FIG. 1 AFTER REASSEMBLY

\begin{tabular}{lrrr}
\hline Parameter & Initial $\mathbf{p}_{0}$ & Optimized $\mathbf{p}^{*}$ & Change $(\%)$ \\
\hline$L_{1}(\mu \mathrm{H})$ & 126. & 126. & 0.079 \\
$L_{2}(\mu \mathrm{H})$ & 68.9 & 68.6 & -0.38 \\
$L_{3}(\mu \mathrm{H})$ & 45.3 & 45.4 & 0.21 \\
$L_{4}(\mu \mathrm{H})$ & 17.8 & 17.9 & 0.67 \\
\hline$k_{12}(\%)$ & 20.5 & 20.1 & -2.2 \\
$k_{13}(\%)$ & 9.90 & 8.12 & -18. \\
$k_{14}(\%)$ & 8.18 & 6.40 & -22. \\
$k_{23}(\%)$ & 7.72 & 6.67 & -14. \\
$k_{24}(\%)$ & 7.59 & 7.13 & -6.1 \\
$k_{34}(\%)$ & 34.7 & 33.6 & -3.4 \\
\hline$R_{1}(\mathrm{~m} \Omega)$ & 142. & 142. & 0.31 \\
$R_{2}(\mathrm{~m} \Omega)$ & 81.3 & 82.9 & 2.0 \\
$R_{3}(\mathrm{~m} \Omega)$ & 66.1 & 71.7 & 8.4 \\
$R_{4}(\mathrm{~m} \Omega)$ & 43.6 & 46.2 & 5.9 \\
\hline
\end{tabular}

TABLE V

TIME AVERAGED MAXIMUM POWER TRANSFER AND EFFICIENCY FOR THE TOP TEN CAPACITANCE COMBINATIONS OF THE CAPACITOR BANKS

\begin{tabular}{llllrl}
\hline $\begin{array}{l}p_{\text {load }} \\
\mathrm{kW}\end{array}$ & \multicolumn{1}{l}{$C_{1}$} & \multicolumn{2}{c}{$C_{2}$} & $C_{3}$ & $C_{4}$ \\
\hline 6.51 & 92.3 & 26.4 & 39.2 & 67.1 & 145 \\
6.48 & 92.2 & 26.4 & 39.2 & 67.1 & 152 \\
6.11 & 92.4 & 26.4 & 39.2 & 67.1 & 139 \\
5.93 & 92.3 & 30.8 & 39.2 & 67.1 & 152 \\
5.91 & 91.3 & 30.8 & 31.3 & 67.1 & 139 \\
\hline 5.84 & 91.8 & 26.4 & 39.2 & 62 & 152 \\
5.75 & 91.6 & 26.4 & 39.2 & 62 & 145 \\
5.74 & 91.3 & 30.8 & 31.3 & 67.1 & 145 \\
$\mathbf{5 . 5 7}$ & $\mathbf{9 1 . 3}$ & $\mathbf{2 6 . 4}$ & $\mathbf{3 9 . 2}$ & $\mathbf{6 2}$ & $\mathbf{1 3 9}$ \\
5.54 & 92.3 & 26.4 & 39.2 & 67.1 & 130 \\
\hline
\end{tabular}

\section{CONCLUSION}

We have presented a system identification and tuning procedure for a wireless power transfer (WPT) system that consists of four magnetically coupled resonators. In the system identification procedure, we employ a Bayesian approach to estimate the self-inductance and losses of the four coils in combination with their magnetic coupling. After the system identification procedure, the WPT model is used to predict appropriate capacitance values for the four capacitor banks to recover high system performance, where the generator voltage is limited by a set of circuit state constraints that prevent component overheating and breakdown. We find that the system identification procedure combined with the four tunable capacitors can account for and take care of rather significant perturbations due to, e.g., manufacturing tolerances and coil displacement.
Further, a large set of different capacitance selections for the four tunable capacitor banks achieved high system performance for an experimentally realized WPT system design with magnetic coupling coefficients on the order of $6 \%$ to $8 \%$ between the primary and secondary side. This feature may be of key importance for future high performance WPT systems in applications where rather low magnetic coupling in combination with large variations in coil positions are expected, such as for electric vehicle charging.

\section{REFERENCES}

[1] S. Li and C. C. Mi, "Wireless Power Transfer for Electric Vehicle Applications," IEEE J. Emerg. Sel. Top. Power Electron., vol. 3, no. 1, pp. 4-17, Mar 2015. [Online]. Available: http://ieeexplore.ieee.org/document/6804648/

[2] F. Musavi and W. Eberle, "Overview of wireless power transfer technologies for electric vehicle battery charging," IET Power Electron., vol. 7, no. 1, pp. 60-66, Jan 2014. [Online]. Available: http://ieeexplore.ieee.org/document/6715805/

[3] B. Esteban, M. Sid-Ahmed, and N. C. Kar, "A Comparative Study of Power Supply Architectures in Wireless EV Charging Systems," IEEE Trans. Power Electron., vol. 30, no. 11, pp. 6408-6422, Nov 2015. [Online]. Available: http://ieeexplore.ieee.org/document/7119591/

[4] A. Kurs, A. Karalis, R. Moffatt, J. D. Joannopoulos, P. Fisher, and M. Soljacic, "Wireless Power Transfer via Strongly Coupled Magnetic Resonances," Science, vol. 317, no. 5834, pp. 83-86, Jul 2007. [Online]. Available: http://www.sciencemag.org/cgi/doi/10.1126/science.1143254

[5] A. P. Sample, D. A. Meyer, and J. R. Smith, "Analysis, Experimental Results, and Range Adaptation of Magnetically Coupled Resonators for Wireless Power Transfer," IEEE Trans. Ind. Electron., vol. 58, no. 2, pp. 544-554, Feb 2011. [Online]. Available: http://ieeexplore.ieee.org/document/5437250/

[6] S. Aldhaher, P. C.-K. Luk, and J. F. Whidborne, "Electronic tuning of misaligned coils in wireless power transfer systems," IEEE Trans. Power Electron., vol. 29, no. 11, pp. 5975-5982, Jan 2014. [Online]. Available: http://ieeexplore.ieee.org/document/6702433/

[7] SAE Standard, "J2954, Wireless Power Transfer for Light-Duty Plug-In/Electric Vehicles and Alignment Methodology," 2016. [Online]. Available: http://standards.sae.org/j2954_201605/

[8] T. C. Beh, M. Kato, T. Imura, S. Oh, and Y. Hori, "Automated impedance matching system for robust wireless power transfer via magnetic resonance coupling," IEEE Trans. Ind. Electron., vol. 60, no. 9, pp. 3689-3698, Sep 2013. [Online]. Available: http://ieeexplore.ieee.org/document/6226848/

[9] S. Li, W. Li, J. Deng, T. D. Nguyen, and C. C. Mi, "A double-sided LCC compensation network and its tuning method for wireless power transfer," IEEE Trans. Veh. Technol., vol. 64, no. 6, pp. 2261-2273, Jun 2015. [Online]. Available: http://ieeexplore.ieee.org/document/6876154/

[10] M. E. Halpern and D. C. Ng, "Optimal tuning of inductive wireless power links: Limits of performance," IEEE Trans. Circuits Syst. I Regul. Pap., vol. 62, no. 3, pp. 725-732, Jan 2015. [Online]. Available: http://ieeexplore.ieee.org/document/7024943/

[11] M. Kiani and M. Ghovanloo, "The Circuit Theory Behind CoupledMode Magnetic Resonance-Based Wireless Power Transmission," IEEE Trans. Circuits Syst. I Regul. Pap., vol. 59, no. 9, pp. 2065-2074, Sep 2012. [Online]. Available: http://ieeexplore.ieee.org/document/6138883/

[12] S. Y. R. Hui, W. Zhong, and C. K. Lee, "A Critical Review of Recent Progress in Mid-Range Wireless Power Transfer," IEEE Trans. Power Electron., vol. 29, no. 9, pp. 4500-4511, Sep 2014. [Online]. Available: http://ieeexplore.ieee.org/document/6472081/

[13] K. Lee and S. H. Chae, "Power Transfer Efficiency Analysis of Intermediate-Resonator for Wireless Power Transfer," IEEE Trans. Power Electron., vol. 8993, no. c, pp. 1-1, Apr 2017. [Online]. Available: http://ieeexplore.ieee.org/document/7913710/

[14] W. Zhong, C. K. Lee, and S. Y. Ron Hui, "General analysis on the use of tesla's resonators in domino forms for wireless power transfer," IEEE Trans. Ind. Electron., vol. 60, no. 1, pp. 261-270, Oct 2013. [Online]. Available: http://ieeexplore.ieee.org/document/6041026/

[15] X. Liu and G. Wang, "A Novel Wireless Power Transfer System with Double Intermediate Resonant Coils," IEEE Trans. Ind. Electron., vol. 63, no. 4, pp. 2174-2180, Dec 2016. [Online]. Available: http://ieexplore.iee.org/document/7362019/ 
[16] D. Lin, J. Yin, and S. Y. R. Hui, "Parameter identification of wireless power transfer systems using input voltage and current," Energy Convers. Congr. Expo. (ECCE), 2014 IEEE, pp. 832-836, Nov 2014. [Online]. Available: http://ieeexplore.ieee.org/document/6953483/

[17] J. Winges, T. Rylander, T. McKelvey, C. Petersson, C. Ekman, and L. Å. Johansson, "System identification and tuning of wpt systems," in Proc. EEEIC/I\&CPS Conf., Jun 2017, pp. 1-5. [Online]. Available: http://ieeexplore.ieee.org/document/7977544/

[18] S. M. Kay and M. K. Steven, Fundamentals of Statistical Signal Processing: Estimation Theory. Englewood Cliffs, NJ, USA: PrenticeHall, Inc., 1993.

[19] COMSOL AB, "COMSOL Multiphysics® v. 5.2," Stockholm, Sweden, 2016. [Online]. Available: www.comsol.com

[20] The MathWorks Inc., "Matlab®," Natick, Massachusetts, United States, 2017. [Online]. Available: www.mathworks.com

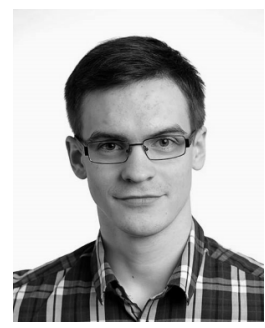

Johan Winges received the B.S. degree in engineering physics from Chalmers University of technology, Gothenburg, Sweden, in 2009, the M.S. degree in applied physics from Chalmers University of Technology in 2011, and the Ph.D. degree in electrical engineering from Chalmers University of Technology in 2016.

$\mathrm{He}$ is currently a researcher at Chalmers University of Technology in the Signal processing group at the Department of Electrical Engineering. His research interests are computational electromagnetics, inverse scattering problems and wireless power transfer.

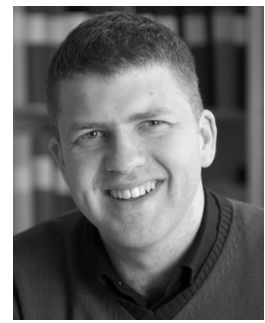

Thomas Rylander received the M.S. degree in electrical engineering from the KTH Royal Institute of Technology, Stockholm, Sweden, in 1997, and the $\mathrm{Ph} . \mathrm{D}$. degree in electrical engineering from Chalmers University of Technology, Göteborg, Sweden, in 2002.

He was a Post-Doctoral Associate with the Center for Computational Electromagnetics, University of Illinois at Urbana-Champaign, Champaign, IL, USA, from 2002 to 2004. He was an Assistant Professor and an Associate Professor with Chalmers University of Technology in 2004 and 2007, respectively. His research interests are electromagnetic field theory with a broad range of applications. In particular, his research is based on computational electromagnetics with a focus on finiteelement-based methods.

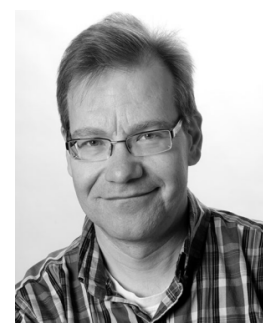

Tomas McKelvey received the M.S. degree in electrical engineering from Lund University, Lund, Sweden, in 1991, and the Ph.D. degree in automatic control from Linköping University, Linköping, Sweden, in 1995.

He has held research and teaching positions with Linköping University from 1995 to 1999 , where he became a Docent in 1999. From 1999 to 2000, he was a Visiting Researcher with The University of Newcastle, Newcastle, NSW, Australia. Since 2000, he has been with the Chalmers University of Technology, Gothenburg, Sweden, where he has been a Full Professor since 2006. $\mathrm{He}$ has been the Head of the Signal Processing Group, Chalmers University of Technology, since 2011. His current research interests include modelbased and statistical signal processing, system identification, machine learning, image processing and control with applications to biomedical engineering, and combustion engines. 